\title{
Cavity QED quantum phase gates for a single longitudinal mode of the intracavity field
}

\author{
R. García-Maraver, ${ }^{1}$ R. Corbalán, ${ }^{1}$ K. Eckert, ${ }^{2}$ S. Rebić, ${ }^{3}$ M. Artoni,,${ }^{4,5}$ and J. Mompart ${ }^{1, *}$ \\ ${ }^{1}$ Departament de Física, Universitat Autònoma de Barcelona, E-08193, Bellaterra, Spain \\ ${ }^{2}$ Institut of Theoretical Physics, University of Hannover, Appelstrasse 2, D-30167, Hannover, Germany \\ ${ }^{3}$ INFM and Department of Physics, Università di Camerino, 62032 Camerino, Italy \\ ${ }^{4}$ Department of Chemistry and Physics of Materials, Via Valotti 9, 25133 Brescia, Italy \\ ${ }^{5}$ European Laboratory for Non-Linear Spectroscopy, Via N. Carrara 1, 50019 Sesto Fiorentino, Italy
}

(Received 29 July 2004; published 30 December 2004)

\begin{abstract}
A single three-level atom driven by a longitudinal mode of a high- $Q$ cavity is used to implement two-qubit quantum phase gates for the intracavity field. The two qubits are associated with the zero- and one-photon Fock states of each of the two opposite circular polarization states of the field. The three-level atom mediates the conditional phase gate provided the two polarization states and the atom interact in a V-type configuration and the two-photon resonance condition is satisfied. Microwave and optical implementations are discussed with gate fidelities being evaluated against several decoherence mechanisms such as atomic velocity fluctuations or the presence of a weak magnetic field. The use of coherent states for both polarization states is investigated to assess the entanglement capability of the proposed quantum gates.
\end{abstract}

DOI: 10.1103/PhysRevA.70.062324

PACS number(s): 03.67.Mn, 42.50.Pq, 32.80.-t

\section{INTRODUCTION}

Throughout the last decade, cavity quantum electrodynamics (CQED) [1-10] in both the microwave and optical regimes has been used to test the most striking quantum features of single atoms interacting with zero-, one-, or fewphoton states. Some relevant examples are the observation of photon trapping states and sub-Poissonian statistics in the micromaser [1], the generation of photon number states on demand [2], the single-atom microlaser [3], nonlinear optics with single atoms and photons [4], and nonclassical statistics in wave-particle quantum correlations [5]. In particular, a series of seminal papers by Haroche and co-workers [6-10] have shown that CQED with long-lived atomic Rydberg states provides one of the simplest systems to unambiguously test the nonlocal nature of quantum mechanics. In all these experiments, the Rabi oscillations between a (vacuum) quantum field and an effective two-level atom were used to entangle the cavity field with the atom. Moreover, the passage of subsequent atoms through the microwave cavity with well controlled velocities was used to entangle them via either real [6] or virtual photons [10] allowing, therefore, the creation of massive Einstein-Podolsky-Rosen pairs.

CQED devices hold great promise as basic tools for quantum networks [11] since they provide an interface between computation and communication, i.e., between atoms and photons. In this context, it is a very important task to look for techniques to quantum engineer the state of the intracavity field. Very recently the use of a three-level atom in a cascade configuration [12] has been suggested to entangle two different longitudinal modes of the radiation field in one single step. In particular, single- and two-bit quantum gates were discussed with the number of photons $(n=0$ or 1$)$ of each mode being the quantum bit of information. Although this

\footnotetext{
*Electronic address: jordi.mompart@uab.es
}

proposal is very interesting its eventual implementation presents two main drawbacks: (i) it requires a high- $Q$ cavity that sustains two different longitudinal radiation modes; and (ii) both modes must be adjusted to very particular frequencies: one mode must be on resonance with one of the bare atomic transition frequencies while the second one must be tuned to one of the dressed states built up by the first longitudinal mode. We note here that three- and multilevel atoms have been extensively investigated in the past as a successful tool for many quantum optics applications [13] and, in particular, for quantum information [4,14-17].

In this paper, we propose a scheme that, while also using a three-level atom, overcomes the disadvantages of the previously discussed proposal [12]. We make use of a single longitudinal mode of the cavity to implement a quantum phase gate (QPG) between the two qubits associated with the zero- and single-photon states of the two opposite circular polarization states of this mode. A precise control of the interaction time between the three-level atom and the mode will yield the conditional evolution needed to implement the QPG, provided that the atom and the two polarization states interact in the so-called V-type configuration and that the two-photon resonance condition is satisfied.

The paper is organized as follows. In Sec. II we briefly review the interaction of a single three-level atom with a few-photon field, discuss the basic ideas of the QPG implementation, and determine the explicit conditions for its realization. In Sec. III we address some practical considerations for the physical implementation of QPGs in both microwave and optical regimes. The application of the QPG to the intracavity field with both circular polarizations in a coherent state is discussed in Sec. IV as a method to entangle the intracavity field. Finally, we summarize the proposal and present the main conclusions in Sec. V.

\section{MODEL}

The model we use in this paper is sketched in Fig. 1(a) and consists of a high- $Q$ cavity with a single longitudinal 


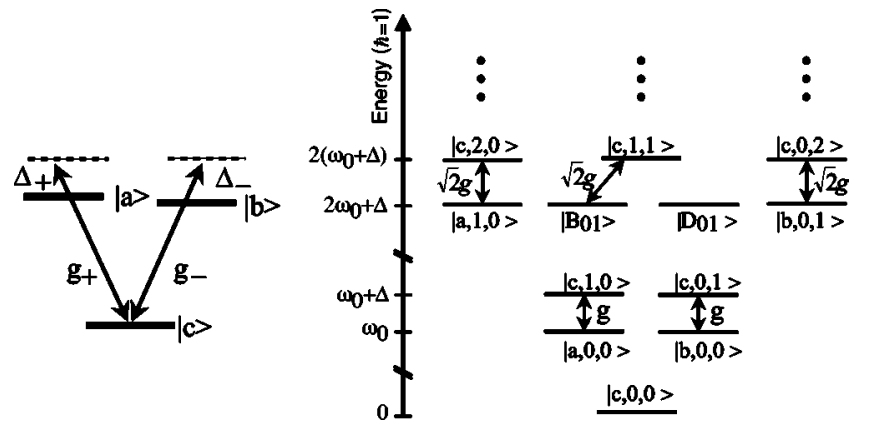

FIG. 1. (a) V-type three-level configuration under investigation. Here $g_{+}\left(g_{-}\right)$is the vaccuum Rabi frequency of the coupling between the atom and the right- (left-)hand circularly polarized field, and $\Delta_{+}\left(\Delta_{-}\right)$is the corresponding cavity-transition detuning. (b) Dressedstate picture of (a) for $g_{+}=g_{-}(\equiv g), \omega_{a c}=\omega_{b c}\left(\equiv \omega_{0}\right)$, and $\Delta+=\Delta-$ $(\equiv \Delta) .\left|B_{01}\right\rangle\left(\left|D_{01}\right\rangle\right)$ is the bright (dark) state combination of $|a, 0,1\rangle$ and $|b, 1,0\rangle$ (see text).

mode at angular frequency $\omega_{c}$ and a three-level atom with its two allowed transition frequencies denoted by $\omega_{a c}$ and $\omega_{b c}$. These two atomic transitions couple to the longitudinal cavity mode via the two opposite circular polarizations $\sigma_{ \pm}$with coupling rates $g_{ \pm}$and detunings $\Delta_{+}=\omega_{c}-\omega_{a c}$ and $\Delta_{-}=\omega_{c}$ $-\omega_{b c}$. For simplicity, in what follows we consider the completely symmetric case given by $g_{+}=g_{-} \equiv g$ and $\Delta_{+}=\Delta_{-} \equiv \Delta$. Later on, in Sec. III, we address the case where $\Delta_{+} \neq \Delta_{-}$due to the Zeeman effect caused by a stray magnetic field.

\section{A. Setting up the idea}

It is very well known [18] that under the two-photon resonance condition the V-type system under investigation can be appropriately described in the bright-dark states basis where the ground state $|c\rangle$ couples only to a particular combination of the atomic bare states $|a\rangle$ and $|b\rangle$, namely, the bright state $|B\rangle$, while remaining uncoupled to the orthogonal superposition, i.e., the dark state $|D\rangle$. In this case, the three-level atom in interaction with the two polarization modes becomes effectively a two-level system, i.e., the atom exhibits Rabi oscillations between the atomic ground state $|c\rangle$ and the bright state $|B\rangle$. Thus, a single complete Rabi oscillation takes the system back to the initial state with a global phase that depends on the detuning. On resonance a $\pi$ phase is attained. These features of the V-type three-level system will be used later on to quantum engineer the intracavity field.

In what follows we define a qubit by the vacuum and single-photon Fock states. Thus, a single longitudinal cavity mode can hold two qubits, one for the right-hand $\left(\sigma_{+}\right)$and one for the left-hand $\left(\sigma_{-}\right)$circular polarization. To drive the conditional evolution between these two qubits, a single three-level atom is initially prepared in the internal ground state $|c\rangle$ and then the interaction is switched on for a controlled period of time. The particular mechanism to switch on and off the interaction will depend on the physical implementation and, accordingly, is discussed in the next section. Using the notation $|i\rangle \otimes|j\rangle \otimes|k\rangle \equiv|i, j, k\rangle$ where, respectively, $i$ denotes the atomic state while $j$ and $k$ denote the number of $\sigma_{+^{-}}$and $\sigma_{-}$-polarized photons, the final state of the system after the interaction can be written, in general, as [see Fig. 1(b)]

$$
\begin{aligned}
& \text { Input state } \text { Output state } \\
& \qquad \begin{aligned}
&|c, 0,0\rangle \rightarrow|c, 0,0\rangle, \\
&|c, 1,0\rangle \rightarrow c_{10}|c, 1,0\rangle+a_{00}|a, 0,0\rangle, \\
&|c, 0,1\rangle \rightarrow c_{01}|c, 0,1\rangle+b_{00}|b, 0,0\rangle, \\
&|c, 1,1\rangle \rightarrow c_{11}|c, 1,1\rangle+B_{01}\left|B_{01}\right\rangle,
\end{aligned}
\end{aligned}
$$

where the $c_{i j}, a_{00}, b_{00}$, and $B_{01}$ are probability amplitudes whose explicit value depends on the detuning and the interaction time. For the symmetric case considered here $c_{01}$ $=c_{10}$ and $a_{00}=b_{00} \cdot\left|B_{01}\right\rangle \equiv(1 / \sqrt{2})(|a, 0,1\rangle+|b, 1,0\rangle)\left[\left|D_{01}\right\rangle\right.$ $\equiv(1 / \sqrt{2})(|a, 0,1\rangle-|b, 1,0\rangle)]$ is the bright (dark) state combination of $|a, 0,1\rangle$ and $|b, 1,0\rangle$. For interaction times such that complete Rabi oscillations occur, i.e., times for which the atom is brought back to the internal state $|c\rangle$, only those phases are left whose explicit value depends on the number of oscillations and the cavity detuning. Therefore, looking for interaction times that yield complete Rabi oscillations both when the initial state is $|c, 1,0\rangle$ (and, therefore, also for $|c, 0,1\rangle)$ and when it is $|c, 1,1\rangle$, two different QPGs can be implemented:

$$
\begin{gathered}
\hat{U}_{1}=e^{i \pi \delta_{j 1} \delta_{k 1}}|c, j, k\rangle\langle c, j, k|, \\
\hat{U}_{2}=-e^{i \pi \delta_{j 0} \delta_{k 0}}|c, j, k\rangle\langle c, j, k| .
\end{gathered}
$$

Notice that $\hat{U}_{1}$ introduces only one phase, equal to $\pi$, when the input state is $|c, 1,1\rangle . \hat{U}_{2}$ on the contrary leaves $|c, 0,0\rangle$ unchanged, but introduces a phase $\pi$ to the other three basis states. Each of these two QPGs together with arbitrary single qubit gates [12] for both circular polarization states yields a universal set of quantum gates and, therefore, these two QPGs can be used to entangle the two polarization states.

We want to note that both $\mathrm{V}$ - and $\Lambda$-type three-level atomic configurations are suitable for the proposal here discussed. The additional advantage of using a V-type scheme is that the common state $|c\rangle$ has lower energy than the other two atomic states and can be radiatively stable if it is the ground state.

\section{B. Conditions for the gate operation}

To look for the conditions needed to implement (2) and (3) we start by writing down the Hamiltonian of the system. In the rotating-wave approximation and the interaction picture, the truncated Hamiltonian of the system restricted to the computational basis reads $(\hbar=1)$

$$
\begin{aligned}
H= & g|a, 0,0\rangle\left\langle c, 1,0\left|e^{-i \Delta t}+g\right| b, 0,0\right\rangle\left\langle c, 0,1\left|e^{-i \Delta t}+g\right| a, 0,1\right\rangle \\
& \times\left\langle c, 1,1\left|e^{-i \Delta t}+g\right| b, 1,0\right\rangle\langle c, 1,1| e^{-i \Delta t}+\mathrm{H} . \mathrm{c} . \\
= & g e^{-i \Delta t}|a, 0,0\rangle\left\langle c, 1,0\left|+g e^{-i \Delta t}\right| b, 0,0\right\rangle\langle c, 0,1| \\
& +\sqrt{2} g e^{-i \Delta t}\left|B_{01}\right\rangle\langle c, 1,1|+\text { H.c. },
\end{aligned}
$$


where $g$ is the vacuum Rabi frequency. It will be shown in Sec. III that for a suitably chosen set of parameters the cavity decay and the spontaneous emission in modes other than the cavity mode can be neglected, based on time-scale arguments. Accordingly, we now solve the Schrödinger equation for this Hamiltonian which, in our case, will provide the same information as the density matrix of the corresponding master equation.

It is clear from Eq. (4) that we deal with three uncoupled two-level systems and a simple analytical solution can be obtained by integrating the corresponding Schrödinger equation. In each of these three cases, the probability amplitude of state $|c, j, k\rangle$ evolves in time according to

$$
\begin{aligned}
c_{j k}(t) \equiv\langle c, j, k \mid \psi(t)\rangle= & \frac{e^{i \Delta t / 2}}{2}\left[\left(1-\frac{\Delta}{\Omega_{j k}}\right) e^{i \Omega_{j k} t / 2}\right. \\
& \left.+\left(1+\frac{\Delta}{\Omega_{j k}}\right) e^{-i \Omega_{j k} t / 2}\right],
\end{aligned}
$$

with $\Omega_{01}=\Omega_{10}=\sqrt{4 g^{2}+\Delta^{2}}$ and $\Omega_{11}=\sqrt{8 g^{2}+\Delta^{2}}$, and where we have assumed $c_{j k}(t=0)=1$ for $j, k=0,1$. Note from Eq. (4) that $c_{00} \equiv\langle c, 0,0 \mid \psi(t)\rangle$ does not evolve in time. Therefore, to implement the first gate transformation (2) one needs

$$
\frac{\Delta t}{2}=2 \pi m, \quad \frac{\Omega_{01} t}{2}=2 \pi n, \quad \frac{\Omega_{11} t}{2}=(2 p+1) \pi,
$$

where the integers $m, n$, and $p$ should satisfy the inequality $2 p+1>2 n>2 m \geqslant 0$. Using the definition of $\Omega_{01}$ and $\Omega_{11}$ it follows from Eq. (6) that

$$
(2 p+1)^{2}=8 n^{2}-4 m^{2},
$$

which is a Diophantine-type equation, whereby for a fixed $n$ the value of $m$ is determined through the detuning $\Delta$ according to the relation $\Delta / g=2 m / \sqrt{n^{2}-m^{2}}$. The problem then reduces to finding the $p$ closest to an integer value that satisfies Eq. (7). Similarly, the implementation of the second quantum phase gate (3) requires

$$
\frac{\Delta t}{2}=2 \pi m, \quad \frac{\Omega_{01} t}{2}=(2 n+1) \pi, \quad \frac{\Omega_{11} t}{2}=(2 p+1) \pi,
$$

leading to the equation

$$
(2 p+1)^{2}=2(2 n+1)^{2}-4 m^{2} .
$$

The corresponding inequality reads $2 p+1>2 n+1>2 m \geqslant 0$, while the relation between $m, n$, and the detuning is $\Delta / g$ $=2 m / \sqrt{(2 n+1)^{2}-m^{2}}$. We report in Table I the best numerical solutions of Eqs. (7) and (9) that, in addition, minimize the interaction time $g t$. Note that the values for the detuning and the interaction time given in Table I are made dimensionless through the vacuum Rabi frequency $g$, which means that these results are general in the sense that they do not rely on any specific physical implementation.

In order to check the validity of our proposal, we have numerically integrated the Schrödinger equation for the four different input states in (1) and checked for both QPGs how much the output states deviate in amplitude and phase from the exact phase gate transformation. We have characterized the deviation by the following fidelity:
TABLE I. Best numerical solutions to Eqs. (7) and (9), sorted by the required interaction time. Detuning $\Delta$ and interaction time $t$ are given in units of $g$ and $g^{-1}$, respectively.

\begin{tabular}{cccccc}
\hline \hline Gate & $m$ & $n$ & $p$ & $\Delta / g$ & $g t$ \\
\hline$\hat{U}_{1}$ & 0 & 6 & 7.985 & 0 & 37.7 \\
$\hat{U}_{2}$ & 8 & 10 & 12.01 & 2.353 & 42.73 \\
$\hat{U}_{1}$ & 12 & 15 & 16.993 & 2.667 & 56.55 \\
$\hat{U}_{1}$ & 4 & 12 & 15.992 & 0.707 & 71.09 \\
$\hat{U}_{2}$ & 18 & 21 & 24.005 & 3.062 & 73.88 \\
$\hat{U}_{2}$ & 10 & 15 & 19.007 & 1.689 & 74.41 \\
$\hat{U}_{1}$ & 24 & 28 & 30.996 & 3.328 & 90.61 \\
$\hat{U}_{2}$ & 0 & 14 & 20.066 & 0 & 91.10 \\
$\hat{U}_{1}$ & 25 & 29 & 32.011 & 3.402 & 92.34 \\
$\hat{U}_{2}$ & 16 & 22 & 27.004 & 2.022 & 99.39 \\
\hline \hline
\end{tabular}

$$
F=\left\langle\left.\left.\left|\sum_{j, k=0,1}\right| c_{j k}^{\text {out }}\right|^{2} e^{i \delta \phi_{j k}}\right|^{2}\right\rangle
$$

where $c_{j k}^{\text {out }} \equiv\left\langle c, j, k \mid \psi_{j k}^{\text {out }}\right\rangle, \delta \phi_{j k}$ is the difference between the phase acquired during the gate operation and the exact phase of the gate defined in Eqs. (2) and (3), and $\langle\cdots\rangle$ denotes the average over the four different input states. Figure 2 shows the time evolution of the fidelity for two different values of the cavity detuning: (a) $\Delta=0$, and (b) $\Delta=2.35 \mathrm{~g}$. Fidelities oscillate with peak values close to $F=1$ for the particular interaction time values predicted in Table I.

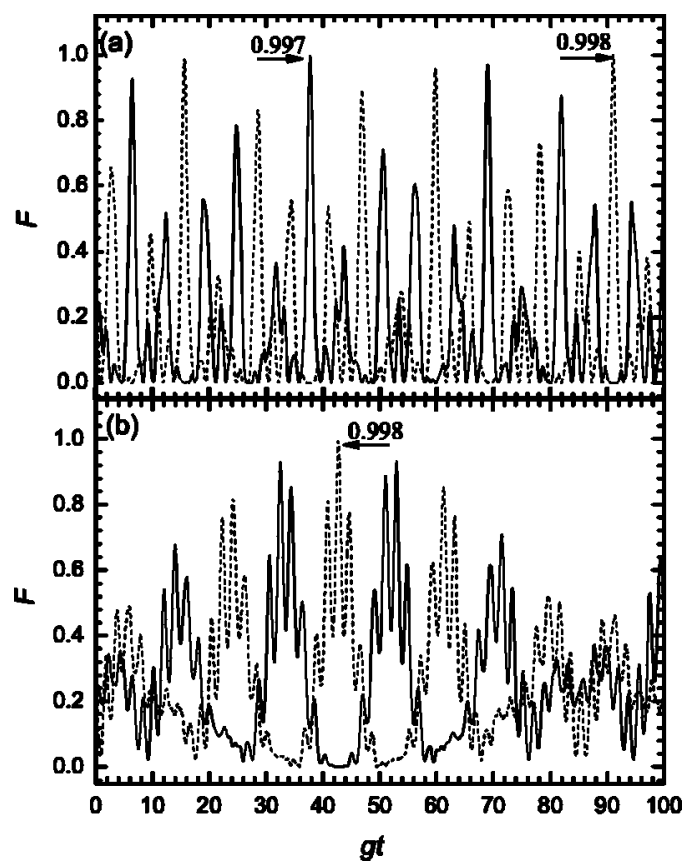

FIG. 2. Time evolution of the fidelity $F$ for both quantum phase gates (solid curve for $\hat{U}_{1}$ and dotted curve for $\hat{U}_{2}$ ). (a) $\Delta=0$, and (b) $\Delta=2.35 \mathrm{~g}$. 


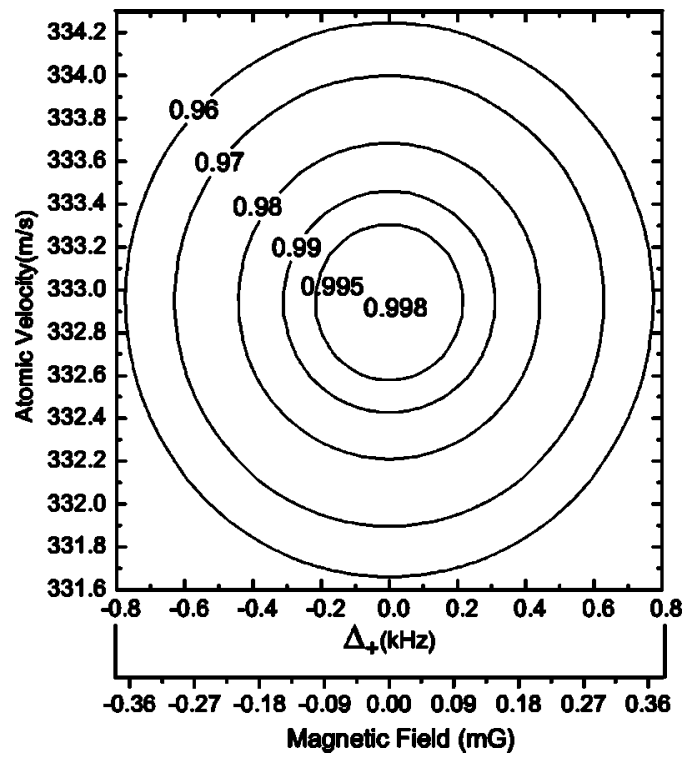

FIG. 3. Fidelity of the first $\hat{U}_{1}$ gate in Table I in the parameter plane atomic velocity versus the intensity of a stray uniform magnetic field along the cavity axis. The parameter settings are $\Delta$ $=0, g=2 \pi \times 50 \mathrm{kHz}$, and an effective cavity length of $L=4 \mathrm{~cm}$.

\section{PHYSICAL IMPLEMENTATIONS}

Up to this point we have discussed the realization of the QPGs for the intracavity field in general terms. Below we give some practical considerations regarding the physical implementation of the previous ideas in both microwave and optical regimes.

\section{A. Microwave regime}

In the microwave regime, single three-level Rydberg atoms crossing the high- $Q$ cavity could be used to implement the QPGs for the intracavity field. The interaction time can be controlled by an accurate selection of the velocity of the incident atoms. Typically, Rydberg atoms used in CQED yield vacuum-Rabi frequencies on the order of $g / 2 \pi$ $\simeq 50 \mathrm{kHz}$ [6], which would imply gate times of a few tenths of a millisecond for the QPGs discussed in this paper. This time must be compared with the lifetime of a photon in a high- $Q$ microwave cavity, which can be as large as a few milliseconds, and with the atomic lifetime in Rydberg states, which can be tens of milliseconds [6].

We have investigated the robustness of the previously discussed QPGs against some of the experimental imperfections existing in the microwave regime. Figure 3 shows the fidelity for the implementation for the first gate in Table I as a function of both the atomic velocity through the cavity and the intensity of a uniform stray magnetic field along the cavity axis. The magnetic field plays in general a negative role as it breaks the degeneracy between atomic states $|a\rangle$ and $|b\rangle$ via the Zeeman effect, i.e., it yields $\Delta_{+}=-\Delta_{-} \neq 0$, such that the closed two-level picture for the $|c, 1,1\rangle \leftrightarrow\left|B_{01}\right\rangle$ transition is no longer valid. The relationship between the cavity detuning and the strength of the magnetic field shown in Fig. 3 is given by $\hbar \Delta_{+}=\mu_{B} g_{J} m_{J} B$ where $g_{J}$ is the gyromagnetic factor and $\mu_{B}$ is the Bohr magneton. To be specific, the values $m_{J}$ $=1$ and $g_{J}=3 / 2$ corresponding to $J=1, L=1$, and $S=1$ have been chosen.

Thus, gate realizations with fidelities $F>0.99$ demand an accuracy of the atomic velocities on the order of a few tenths of meters per second and magnetic fields smaller than a few tenths of milligauss. Both requirements could be achieved in present CQED experiments with Rydberg atoms [10]. Similar results are obtained for the rest of the QPGs shown in Table I.

\section{B. Optical regime}

By working in the optical regime, all the cryogenic requirements necessary in the microwave case to reduce the thermal photon noise can be avoided. In the optical regime, a possible atomic candidate to drive the QPG in the intracavity field is strontium [19]. Depending on the specific finestructure component and on its four natural isotopes [three of which are bosonic, ${ }^{88} \mathrm{Sr}$ (relative abundance $82 \%$ ), ${ }^{86} \mathrm{Sr}$ $(10 \%)$, and $\left.{ }^{84} \mathrm{Sr}(0.5 \%)\right]$, a wide choice of transitions with different $g$ 's, linewidths, and wavelengths are possible. The intercombination line $5{ }^{1} S_{0}-5{ }^{3} P_{1}$ of ${ }^{88} \mathrm{Sr}$, in particular, spans two transitions that couple the ground state with vanishing nuclear spin with two fairly long-lived $(\tau=20 \mu \mathrm{s})$ degenerate $J=1$ states. They both fall in the visible range $(\lambda=689 \mathrm{~nm})$, and hence are easily accessible with common semiconductor lasers; and both can acquire a large vacuum Rabi frequency $g / 2 \pi \simeq 25 \mathrm{MHz}$ when high-finesse microcavities $(F \simeq 3$ $\left.\times 10^{6}\right)$ are used [20]. The coherent atom-field interaction needs to dominate over decoherence rates; hence the strong coupling regime of CQED is required. For the cavity decay rate $\kappa$ and spontaneous emission rate $\gamma$, this means that $g$ $\gg(\kappa, \gamma)$. As the vacuum Rabi frequency $g$ is not constant throughout the cavity mode volume, optimal results will be obtained for an atom trapped at the antinode of the cavity field. This is experimentally viable, as shown, e.g., in Refs. [21], where trapping times up to $1 \mathrm{~s}$ have been reported in the strong-coupling regime. A possible mechanism to control the interaction time could be to switch off and on a magnetic field that breaks both the one- and two-photon resonance conditions, and therefore inhibits the dynamical evolution of the system. Results shown in Table I suggest that the gate operation can be implemented over times $t_{\text {gate }}$ of the order of $1 \mu \mathrm{s}$. Due to the very high finesse of the microcavities [20], lifetimes $\tau_{c a v}$ of the order of a few microseconds can possibly be achieved, implying $t_{\text {gate }} \leqslant \tau_{\text {cav }}$, which is in turn appreciably less than the atomic natural lifetime $\tau$. For the realization of gate fidelities $F>0.99$, the condition $t_{\text {gate }} \ll \tau_{\text {cav }}$ needs to be satisfied. This is feasible, given the rapid progress of optical microcavities over recent years [20,21].

\section{ENTANGLING THE INTRACAVITY FIELD}

Application of a two-qubit QPG to an intracavity field with well defined photon number clearly produces only a global phase. For quantum computation purposes one also needs to produce single-qubit (local) operations for each polarization. Methods for the implementation of single-qubit 


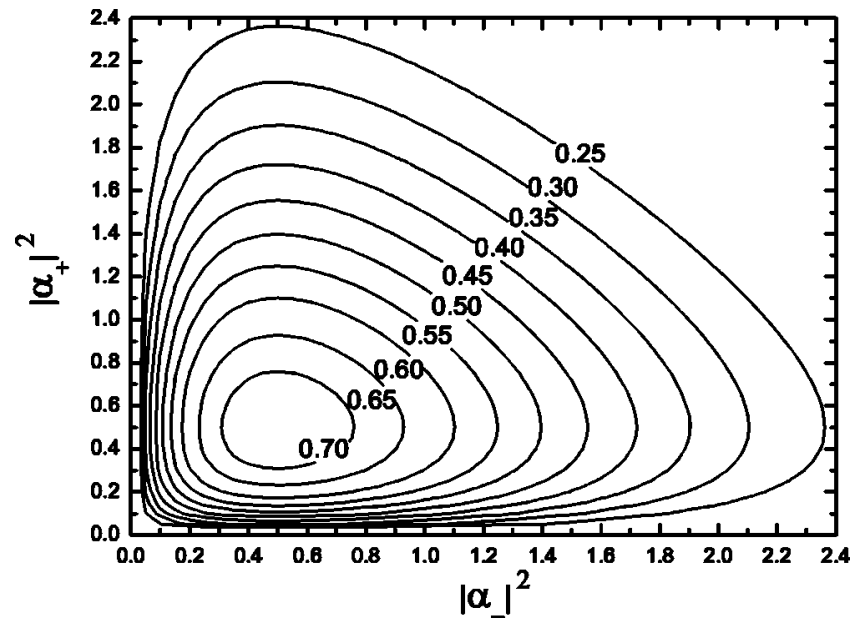

FIG. 4. Concurrence between the two polarization states of the intracavity field (restricted to the computational basis) as a function of the mean photon number of both polarizations. Parameters correspond to the first $\hat{U}_{1}$ gate sorted in Table I.

operations for the intracavity field have been considered in [12]. To only demonstrate the entanglement capability, there is a very simple and accessible approach to entangle the two circular polarizations of the intracavity field. We will not start with a well defined photon number in each mode, but initially inject into the high- $Q$ cavity a product state consisting of a coherent state for both circular polarizations:

$$
\left|\alpha_{+}, \alpha_{-}\right\rangle \equiv e^{-\left|\alpha_{+}\right|^{2} / 2} \sum_{n=0}^{\infty} \frac{\alpha_{+}^{n}}{\sqrt{n !}}|n\rangle \otimes e^{-\left|\alpha_{-}\right|^{2} / 2} \sum_{m=0}^{\infty} \frac{\alpha_{-}^{m}}{\sqrt{m !}}|m\rangle .
$$

Here $\left|\alpha_{+}\right|^{2}\left(\left|\alpha_{-}\right|^{2}\right)$ is the mean photon number of the $\sigma_{+}\left(\sigma_{-}\right)$ polarization. This state is clearly separable. To quantify the entanglement between the two polarization states we use the concurrence [22] $\quad C \equiv 2\left|c_{00} c_{11}-c_{01} c_{10}\right|$, where $c_{j k}$ $\equiv\left\langle c, j, k\left|\hat{U}_{s}\right| \alpha_{+}, \alpha_{-}\right\rangle$where $s=1,2$. In Fig. 4 we show the concurrence after the interaction with the atom as a function of the mean photon number of each polarization. Notice that $C$ takes into account only the Hilbert space of zero and one photon in each polarization mode, while other correlations are ignored. The maximum concurrence $C=0.73$ is reached when the mean photon number of both left and right polarizations is equal to $\langle N\rangle=0.5$.

\section{CONCLUSIONS}

We have proposed a CQED technique to quantum engineer the intracavity field. Single three-level atoms are used to implement two different QPGs between the vacuum and single-photon states of the two opposite circular polarizations of a single longitudinal mode. QPG realizations with fidelities above 0.99 and gate times of around $0.1 \mathrm{~ms}$ for the microwave regime and a few microseconds for the optical regime can be attained, which lie below achievable photon lifetimes in high- $Q$ cavities. However, to evaluate the fidelity also in the case where the gate times are closer to the cavity lifetime, the cavity decay should be included explicitly in our calculations. Some practical considerations such as the role of atomic velocity fluctuations or the presence of a uniform stray magnetic field along the cavity axis have been addressed, showing that the QPGs here discussed can be implemented with state of the art technology. We have applied a QPG in the case where each circular polarization of the intracavity field is initially in a coherent state. This, as has been demonstrated, constitutes a simple method to entangle the two polarization states of the intracavity field. We want to note that the ideas here discussed to quantum engineer the intracavity field could be extended to other CQED physical systems of current interest, e.g., solid state devices [23], such as superconducting electrical circuits, and superconducting quantum interference devices [24,25]. Finally, we would like to note also that the use of three-level atoms in the optical regime has been previously discussed in the CQED literature $[4,15]$. For instance, single three-level atoms in a $\Lambda$-type configuration have been used recently to deterministically produce a single-photon source [15].

\section{ACKNOWLEDGMENTS}

We gratefully acknowledge enlightening discussions with G. Ferrari, J. Raimond, and D. Vitali. Financial support comes from the MCyT (Spanish Government) under contracts BFM2002-04369-C04-02 and HI2003-0075 (Acción Integrada España-Italia), from the DGR (Catalan Government) under contract 2001SGR00187, by the Deutsche Forschungsgemeinschaft through the research program "Quanteninformationsverarbeitung," and by the Ministerio dell'Istruzione, dell'Università e della Ricerca (MIUR) under Contract IT1603 (Azione Integrada Italia-Spagna). R. G.-M. acknowledges support from the MCyT (Grant No. BES2003-1765)
[1] H. Walther, Phys. Rep. 219, 263 (1992).

[2] H. Walther, Fortschr. Phys. 51, 521 (2003).

[3] K. An, J. J. Childs, R. R. Dasari, and M. S. Feld, Phys. Rev. Lett. 73, 3375 (1995); K. An and M. S. Feld, Phys. Rev. A 56, 1662 (1997).

[4] Q. A. Turchette, C. J. Hood, W. Lange, H. Mabuchi, and H. J. Kimble, Phys. Rev. Lett. 75, 4710 (1995).

[5] G. T. Foster, L. A. Orozco, H. M. Castro-Beltran, and H. J.
Carmichael, Phys. Rev. Lett. 85, 3149 (2000).

[6] E. Hagley et al., Phys. Rev. Lett. 79, 1 (1997).

[7] X. Maitre et al., Phys. Rev. Lett. 79, 769 (1997).

[8] A. Rauschenbeutel et al., Phys. Rev. Lett. 83, 5166 (1999).

[9] G. Nogues et al., Nature (London) 400, 239 (1999).

[10] S. Osnaghi et al., Phys. Rev. Lett. 87, 037902 (2001).

[11] J. I. Cirac et al., Phys. Scr., T 76, 223 (1998).

[12] M. S. Zubairy, M. Kim, and M. O. Scully, Phys. Rev. A 68, 
033820 (2003).

[13] J. Mompart and R. Corbalán, J. Opt. B: Quantum Semiclassical Opt. 2, R7 (2000).

[14] M. D. Lukin and A. Imamoglu, Phys. Rev. Lett. 84, 1419 (2000); Nature (London) 413, 273 (2001).

[15] M. Hennrich, T. Legero, A. Kuhn, and G. Rempe, Phys. Rev. Lett. 85, 4872 (2000).

[16] A. Biswas and G. S. Agarwal, Phys. Rev. A 69, 062306 (2004).

[17] C. Ottaviani, D. Vitali, M. Artoni, F. Cataliotti, and P. Tombesi, Phys. Rev. Lett. 90, 197902 (2003); S. Rebić et al., Phys. Rev. A 70, 032317 (2004).

[18] E. Arimondo, in Progress in Optics, edited by E. Wolf (Elsevier Science, Amsterdam, 1996), Vol. 35, p. 257.

[19] M. Takamoto and H. Katori, Phys. Rev. Lett. 91, 223001
(2003); G. Ferrari et al., ibid. 91, 243002 (2003); H. Katori, T. Ido, Y. Isoya, and M. Kuwata-Gonokami, ibid. 82, 1116 (1999).

[20] G. Rempe et al., Opt. Lett. 17, 363 (1992).

[21] J. McKeever et al., Phys. Rev. Lett. 90, 133602 (2003); J. A. Sauer, K. M. Fortier, M. S. Chang, C. D. Hamley, and M. S. Chapman, Phys. Rev. A 69, 051804(R) (2004).

[22] S. Hill and W. K. Wootters, Phys. Rev. Lett. 78, 5022 (1997).

[23] G. Khitrova, H. M. Gibbs, F. Jahnke, M. Kira, and S. W. Koch, Rev. Mod. Phys. 71, 1591 (1999).

[24] A. Blais, R. S. Huang, A. Wallraff, S. M. Girvin, and R. J. Schoelkopf, Phys. Rev. A 69, 062320 (2004).

[25] C.-P. Yang, Shih-I. Chu, and S. Han, Phys. Rev. Lett. 92, 117902 (2004). 